\title{
Bridging spiking neuron models and mesoscopic population models - a general theory for neural population dynamics
}

\author{
Tilo Schwalger*, Moritz Deger, Wulfram Gerstner \\ From 24th Annual Computational Neuroscience Meeting: CNS*2015 \\ Prague, Czech Republic. 18-23 July 2015
}

Many circuits of the brain can be described by a system of interacting neural populations that are approximately homogeneous. For instance, cortical layers typically consist of a few main types of excitatory and inhibitory neurons that form small homogeneous populations of neurons. Such systems can be modeled on different spatial scales. On the microscopic scale, single cell activity has been faithfully described by reduced phenomenological neuron models [1]. Simulations of networks of such neuron models are, however, computationally expensive and do not offer much analytical insight. On the other hand, mesoscopic population models are reduced descriptions of the global activities of each population. These activities are stochastic due to the finite sizes of the populations. Mesoscopic models can be efficiently simulated and provide a better understanding of the dynamics owing to the abstraction of microscopic information. However, it is largely unknown how to relate mesoscopic population models to microscopic properties such as neural refractoriness, synaptic conductance dynamics and spike-frequency adaptation.

Here, we derive a mesoscopic population model for microscopic networks of generalized integrate-and-fire neurons [1]. This type of neuron model supports important properties like neural refractoriness, multiple-timescale adaptation, stochastic spike generation and synaptic dynamics; its parameters can be directly extracted from experiments of real neurons. In particular, we use a meanfield and a quasi-renewal approximation [1] to derive stochastic integral equations for the population rates.

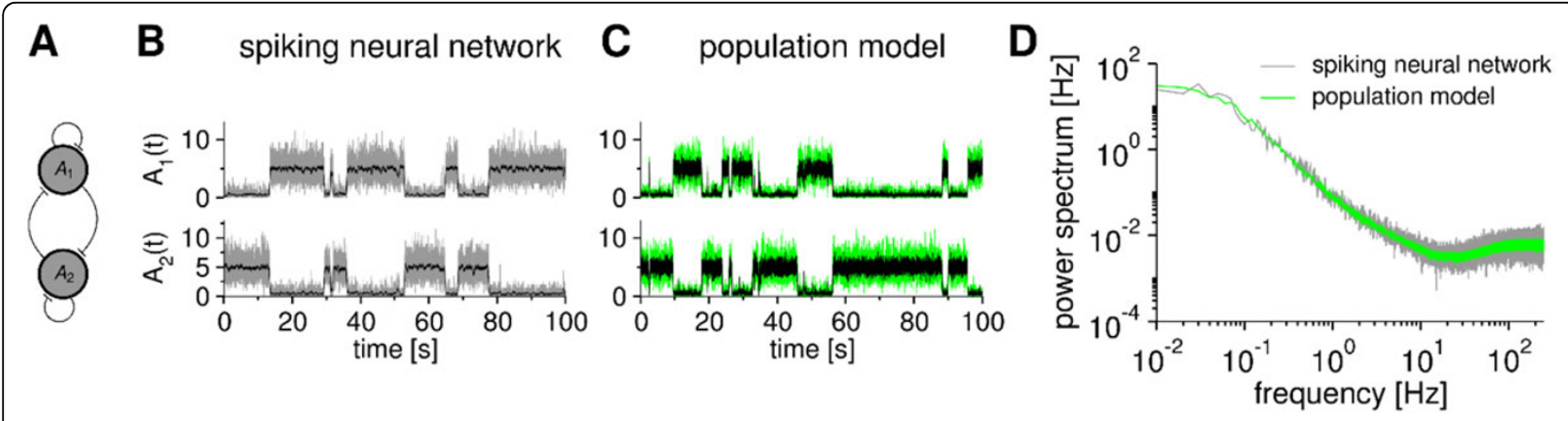

Figure 1 Stochastic population equation precisely captures the collective bistable dynamics of a spiking neural network. A Two mutually inhibitory populations of 500 neurons each. B,C Sample paths of the spiking neural network and the derived population model, respectively. Transitions are due to finite-size fluctuations. D The population activity of microscopic and mesoscopic model have the same firstand second-order statistics.

\footnotetext{
* Correspondence: tilo.schwalger@epfl.ch

Brain Mind Institute, École Polytechnique Fédérale de Lausanne (EPFL)

Station 15, 1015 Lausanne, Switzerland
} 
These equations highlight how the history of activities and fluctuations affects the refractoriness of the populations and the activities at the current time. They can be solved forward in time and thus allow to quickly generate stochastic samples of spontaneous or evoked activities (Fig. 1B,C) that have the same statistics as a corresponding microscopic network simulation to a high degree of accuracy (Fig. 1D). The theory not only captures linear population dynamics [2] but also nonlinear collective effects that emerge on the population level such as metastability (Fig. 1). Our novel theory establishes a general framework for modeling neural population dynamics based on microscopic neuronal parameters. It offers an efficient way to analyze cortical circuits and its computational functions, and how they depend on single-cell and synaptic properties.

\section{Acknowledgements}

Research was supported by the European Research Council (Grant

Agreement no. 268689, MultiRules)

Published: 18 December 2015

\section{References}

1. Gerstner W, Kistler WM, Naud R, Paninski L: Neuronal Dynamics: From Single Neurons to Networks and Models of Cognition Cambridge: Cambridge University Press; 2014.

2. Deger M, Schwalger T, Naud R, Gerstner W: Fluctuations and information filtering in coupled populations of spiking neurons with adaptation. Phys Rev E 2014, 90(6-1):062704

\section{doi:10.1186/1471-2202-16-S1-P79}

Cite this article as: Schwalger et al.: Bridging spiking neuron models and mesoscopic population models - a general theory for neural population dynamics. BMC Neuroscience 2015 16(Suppl 1):P79.

\section{Submit your next manuscript to BioMed Central} and take full advantage of:

- Convenient online submission

- Thorough peer review

- No space constraints or color figure charges

- Immediate publication on acceptance

- Inclusion in PubMed, CAS, Scopus and Google Scholar

- Research which is freely available for redistribution

Submit your manuscript at www.biomedcentral.com/submit
C Biomed Central 